\title{
Corrigendum: Polypharmacy to Mitigate Acute and Delayed Radiation Syndromes
}

\author{
Tracy Gasperetti ${ }^{1 * t}$, Tessa Miller ${ }^{1 \dagger}$, Feng Gao ${ }^{1}$, Jayashree Narayanan ${ }^{1}$, \\ Elizabeth R. Jacobs ${ }^{2,3,4,5}$, Aniko Szabo ${ }^{6}$, George N. Cox ${ }^{7}$, Christie M. Orschell ${ }^{8}$, Brian L. Fish ${ }^{1}$ \\ and Meetha Medhora ${ }^{1,2,3,4,5}$
}

${ }^{1}$ Department of Radiation Oncology, Medical College of Wisconsin, Milwaukee, WI, United States, ${ }^{2}$ Department of Medicine, Medical College of Wisconsin, Milwaukee, WI, United States, ${ }^{3}$ Department of Physiology, Medical College of Wisconsin, Milwaukee, WI, United States, ${ }^{4}$ Cardiovascular Center, Medical College of Wisconsin, Milwaukee, WI, United States, ${ }^{5}$ Department of Veterans Affairs, Research Service, Zablocki VAMC, Milwaukee, WI, United States, ${ }^{6}$ Institute for Health and Equity, Division of Biostatistics, Medical College of Wisconsin, Milwaukee, WI, United States, ${ }^{7}$ Bolder BioTechnology Inc., Boulder, CO, United States, ${ }^{8}$ Department of Medicine, Indiana University School of Medicine, Indianapolis, IN, United States

Keywords: polypharmacy, acute radiation syndrome, delayed effects of acute radiation exposure, mitigation, hematopoietic growth factor, lisinopril, supportive care, radiation pneumonitis

\section{Corrigendum on}

\section{OPEN ACCESS}

Edited and reviewed by: Ann Farese, University of Maryland, Baltimore, United States

${ }^{*}$ Correspondence: Tracy Gasperetti tgasperetti@mcw.edu

${ }^{t}$ These authors have contributed equally to this work and share first authorship

Specialty section: This article was submitted to Translational Pharmacology, a section of the journal

Frontiers in Pharmacology

Received: 14 July 2021 Accepted: 09 August 2021 Published: 25 August 2021

Citation:

Gasperetti T, Miller T, Gao F, Narayanan J, Jacobs ER, Szabo A, Cox GN, Orschell CM, Fish BL and Medhora M (2021) Corrigendum: Polypharmacy to Mitigate Acute and

Delayed Radiation Syndromes.

Front. Pharmacol. 12:741485.

doi: 10.3389/fphar.2021.741485
Polypharmacy to Mitigate Acute and Delayed Radiation Syndromes

by Gasperetti, T., Miller, T., Gao, F., Narayanan, J., Jacobs, E. R., Szabo, A., Cox, G. N., Orschell, C. M., Fish, B, L., and Medhora, M. (2021). Front. Pharmacol. 12:634477. 10.3389/fphar.2021.634477

In the original article, there was a mistake in Figures 2, 4, 9 as published. "The y axis was incorrectly labeled as 0-100, whereas it should be labeled 100-0.” The corrected Figures 2, 4, 9 appear below.

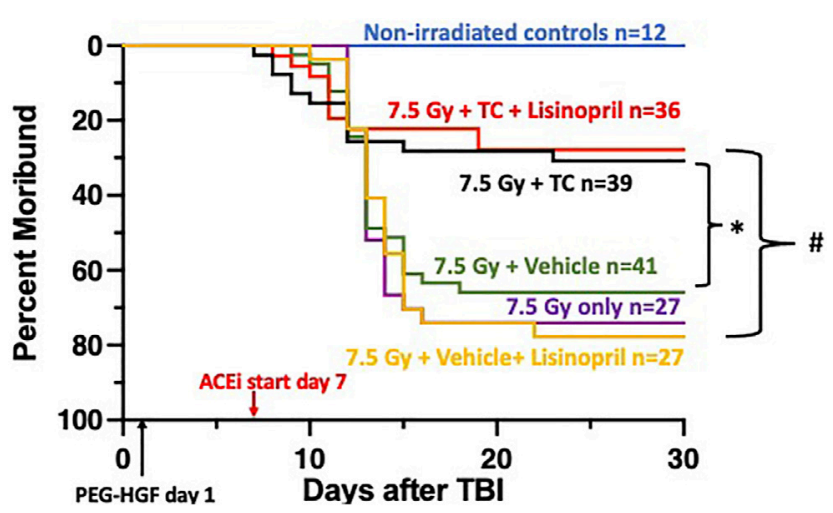

FIGURE 2 | Mitigation of hematopoietic-acute radiation syndrome (H-ARS) by triple combination with and without lisinopril. Kaplan-Meier plots show morbidity through 30-days after 7.5 Gy total body irradiation (TBI). The triple combination (TC, consisting of PEG-hG-CSF, PEG mGM-CSF and PEG hIL-11) or vehicle were given subcutaneously 24-h after TBI (designated by PEG-HGF) and the ACE inhibitor, lisinopril, was started in the drinking water 7 days after irradiation. The number of rats in each group is designated by the "n." Non-irradiated controls are represented with the blue line. Morbidity was not different in the three irradiated groups given 7.5 Gy only, with vehicle or lisinopril, but survival was enhanced in the group which received the TC ( 0.05 , denoted by * compared to $7.5 \mathrm{~Gy}+$ vehicle group). Survival was increased in the irradiated group receiving TC and lisinopril compared to the irradiated rats receiving the vehicle for TC and lisinopril $(p<0.05$, denoted by \#). 


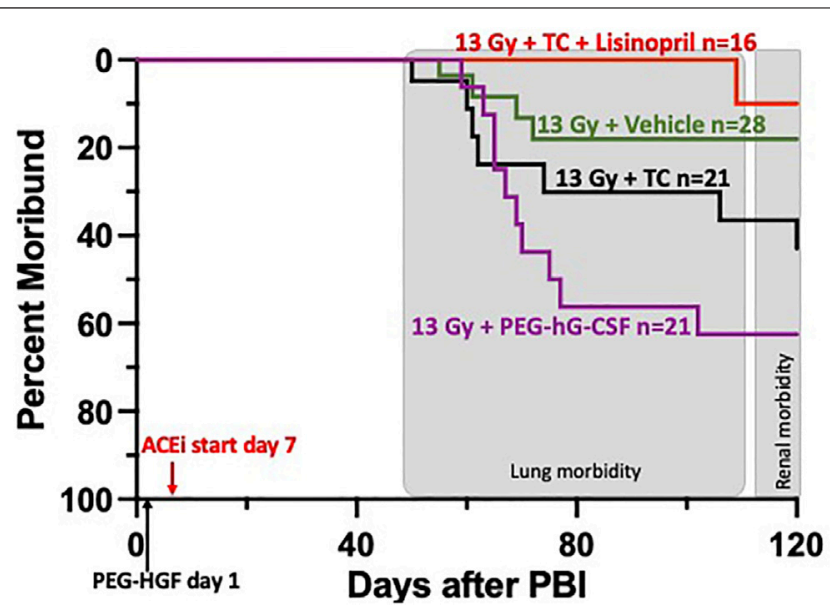

FIGURE 4 | Kaplan-Meier plot representing morbidity from DEARE up to 120 days post 13 Gy partial body irradiation with one hind limb shielded (legout PBI). The triple combination (TC, consisting of PEG-hG-CSF, PEG mGMCSF and PEG hlL-11), vehicle or PEG-hG-CSF (BBT-015) were given subcutaneously 24 h post leg-out $\mathrm{PBI}$ (designated by PEG-HGF) and the ACE inhibitor, lisinopril, was started in the drinking water 7 days post irradiation $\left(24 \mathrm{mg} \mathrm{m}^{-2} \mathrm{~d}^{-1}\right)$. All irradiated rats were given supportive care with subcutaneous hydration $\left(40 \mathrm{ml} \mathrm{kg}^{-1} \mathrm{~d}^{-1}\right)$ and enrofloxacin $\left(10 \mathrm{mg} \mathrm{kg}^{-1} \mathrm{~d}^{-1}\right)$ from days $3-7$ to $2-14$, respectively,. There was trend in lower morbidity in irradiated rats that received TC + lisinopril compared to irradiated rats that received PEG-hG-CSF, but this was not significant ( $p$ 0.07). Shaded (gray) areas represent the timing for the lung and renal injuries.

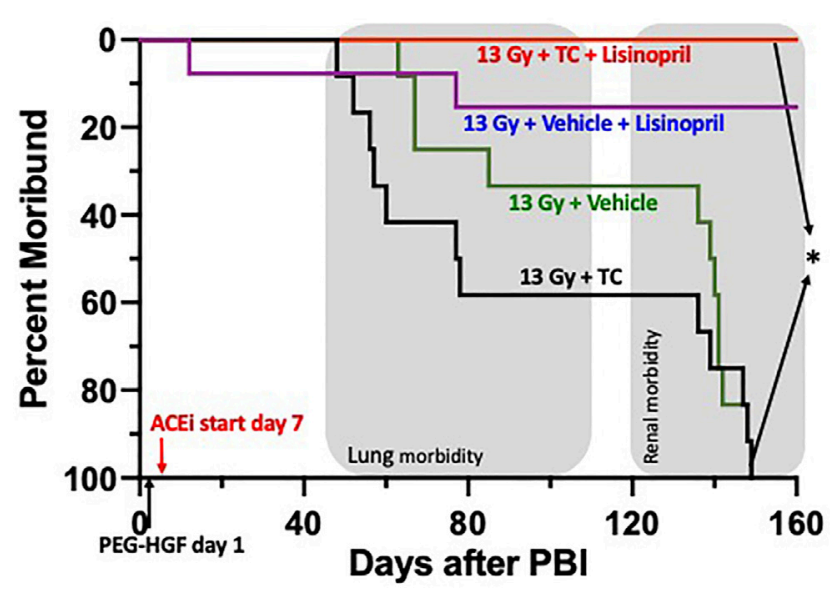

FIGURE 9 | Kaplan-Meier plot representing morbidity from DEARE up to 160 days post 13 Gy partial body irradiation with one hind limb shielded (legout PBI). The triple combination (TC, consisting of PEG-hG-CSF, PEG mGMCSF and PEG hlL-11), or vehicle were given subcutaneously 24 h post PBI and the ACE inhibitor, lisinopril, was started in the drinking water 7 -days post-irradiation. All irradiated rats were given supportive care. All irradiated rats that received TC + lisinopril survived to 160 days as compared to $100 \%$ morbidity for irradiated rats that received TC alone $(p<0.0001)$. Survival of irradiated rats given vehicle + lisinopril was over $90 \%$, while irradiated rats given only the vehicle were moribund before 160 days. Shaded (gray) areas represent the timing for the lung and renal injuries.

The authors apologize for this error and state that this does not change the scientific conclusions of the article in any way. The original article has been updated.

Conflict of Interest: The authors declare that the research was conducted in the absence of any commercial or financial relationships that could be construed as a potential conflict of interest.

Publisher's Note: All claims expressed in this article are solely those of the authors and do not necessarily represent those of their affiliated organizations, or those of the publisher, the editors and the reviewers. Any product that may be evaluated in this article, or claim that may be made by its manufacturer, is not guaranteed or endorsed by the publisher.

Copyright (C) 2021 Gasperetti, Miller, Gao, Narayanan, Jacobs, Szabo, Cox, Orschell, Fish and Medhora. This is an open-access article distributed under the terms of the Creative Commons Attribution License (CC BY). The use, distribution or reproduction in other forums is permitted, provided the original author(s) and the copyright owner(s) are credited and that the original publication in this journal is cited, in accordance with accepted academic practice. No use, distribution or reproduction is permitted which does not comply with these terms. 